\title{
Interactive Radio Experiences
}

\author{
Rik Bauwens \\ VRT \\ Brussels, Belgium \\ rik.bauwens@vrt.be
}

\section{Iris Jennes}

IMEC

Brussels, BE

Iris.Jennes@imec.be

\author{
Hendrik Lievens, Maarten Wijnants \\ Hasselt University - tUL \\ Expertise Centre for Digital Media \\ Hasselt, Belgium \\ hendrik.lievens@uhasselt.be \\ maarten.wijnants@uhasselt.be
}

\author{
Chris Pike \\ BBC \\ Salford, UK \\ chris.pike@bbc.co.uk
}

\section{Werner Bailer}

JOANNEUM RESEARCH

Graz, Austria

werner.bailer@joanneum.at

\section{ABSTRACT}

Nowadays radio shows are much more than a linear broadcast feed - they are all about user engagement. At the same time, many users are no longer only connected to a radio station brand through the linear broadcast channel, but also through digital platforms, and interaction via social media is becoming ever more important. Additionally, digital services enable broadcasters and users to customise the radio experience. Radio is thus a medium embedded in a context of social media, interaction and

Permission to make digital or hard copies of part or all of this work for personal or classroom use is granted without fee provided that copies are not made or distributed for profit or commercial advantage and that copies bear this notice and the full citation on the first page. Copyrights for third-party components of this work must be honored. For all other uses, contact the owner/author(s)

TVX '19, June 5-7, 2019, Salford (Manchester), United Kingdom

(C) 2019 Copyright held by the owner/author(s).

ACM ISBN 978-1-4503-6017-3/19/06.

https://doi.org/10.1145/3317697.3323347 
personalisation. This workshop thus aims to bring together researchers and practitioners working on tools, services and applications enabling interactive radio experiences.

\section{CCS CONCEPTS}

- Information systems $\rightarrow$ Multimedia content creation; • Human-centered computing $\rightarrow$ Interaction paradigms.

\section{KEYWORDS}

radio, interaction; user engagement; object-based media

\section{ACM Reference Format:}

Rik Bauwens, Hendrik Lievens, Maarten Wijnants, Iris Jennes, Chris Pike, and Werner Bailer. 2019. Interactive Radio Experiences. In ACM International Conference on Interactive Experiences for TV and Online Video (TVX '19), June 5-7, 2019, Salford (Manchester), United Kingdom. ACM, New York, NY, USA, 6 pages. https://doi.org/10.1145/ 3317697.3323347

\section{AIMS AND SCOPE}

Nowadays radio shows are much more than a linear broadcast feed - they are all about user engagement. At the same time, many users are no longer only connected to a radio station brand through the linear broadcast channel, but also through digital platforms, and interaction via social media is becoming ever more important. Additionally, digital services enable broadcasters and users to customise the radio experience. Radio is thus a medium embedded in a context of social media, interaction and personalisation. For this reason, radio is not only about audio and music, but also about the text, image and video content that is exchanged as part of the interactions in contemporary radio consumption practices.

Despite the increasingly interactive nature of the radio experience, this topic has been neglected so far at TVX and other conferences addressing interactive media. This workshop thus aims to bring together researchers and practitioners working on tools, services and applications enabling interactive radio experiences.

Relevant topics include (but are not limited to):

- Personalisation of the radio broadcast signal

- Creating interaction bridges between radio creators and consumers

- Use of different media to complement and augment the radio experience

- Steps towards more immersive radio experiences

- Use of object-based audio for personalised and immersive audio presentation

- Using smart speakers (which may include screens in future versions) in the context of radio consumption 
- The potential role of radio in Augmented and Virtual Reality (AR/VR) setups

- Discovery and recommendation systems for radio and audio content

- Big data analysis for profiling listeners and customising radio experiences

- Technological support enabling new radio formats and programmes

We are interested to receive both academic and industrial contributions related to these topics. While demonstration of results and outcomes of experiments and validation are considered relevant for the workshop, this does not exclude submissions at earlier stages. That being said, the focus of this workshop is on contributions that have at least some technological support. Stated differently, we are not targeting submissions that are purely conceptual in nature or that are still residing in an ideation phase. Also of interest are contributions that describe approaches that did not work out, gaps in the state of the art that have been identified and lessons learned from creating (tools for) interactive radio experiences.

The expected outcome of the workshop is a set of emerging topics and research collections related to interactive radio experiences, as mustered in the discussion session of the workshop. The outcome will be documented as a poster to be presented in the main conference. In addition, the aim is to also create a more detailed write-up of the results, for example in the form of a journal paper.

The H2020 MARCONI project, in which four workshop organizers are directly involved, will run open pilots in late 2019. When feasible, selected contributors of submissions presented at the workshop could be invited to take part in those pilots.

\section{FORMAT AND SCHEDULE}

The workshop is planned as a half day event. The workshop format is chosen to present and challenge different views around the topic of interactive radio experiences from both industry and academia. The workshop will contain a discussion round with the aims to identify innovation opportunities, new research directions and open challenges. Those findings will be collected and organised in a wrap-up section (see below), and will serve as source for the documentation and write-up of the workshop.

Introduction (30min). A presentation given jointly by the workshop organisers to outline the scope of the workshop and give a brief overview of the research projects that triggered the ideas for organising the workshop (see also the "Plans for dissemination" Section further down). This presentation aims (a) to be an orientation for workshop participants who are new to the topic, and (b) to tune the mindset of seasoned radio practitioners and researchers to the scope and objectives of the workshop.

Invited talk (30min). Invited talk from the industry presenting exemplary work in the context of interactive radio experiences. 
${ }^{1}$ https://en.wikipedia.org/wiki/Fishbowl_ (conversation)

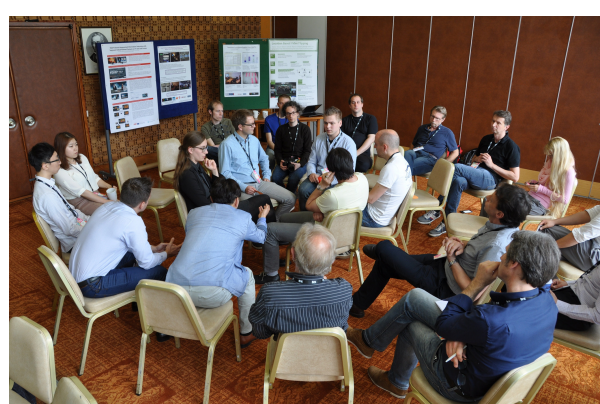

Fishbowl discussions have been successfully used in the WSICC workshop series [1] at previous TVX editions.

\section{Sidebar 1: Fishbowl at WSICC 2014 photo by Rene Kaiser.}

\footnotetext{
${ }^{2}$ https://www.projectmarconi.eu
}

${ }^{3}$ https://www.hradio.eu/
Presentations I (45min). A block of three short oral presentations. We will call for papers and practitioner contributions (see below), and we aim to alternate these two types of presentations rather than having one "academic" and one "industry" block of presentations. Presenters are encouraged to show materials from pilots and trials and focus on the lessons learned.

\section{Presentations II (45min). Same format as Presentations I.}

Discussion (60min). Discussion about the inputs from the presentations, and inputs from other workshop participants with the aim to identify research directions related to interactive radio experiences and to define a set of future opportunities as well as challenges. A discussion format such as fishbowl ${ }^{1}$, which fosters active involvement of all workshop participants in the discussion, will be used. As such, the discussion part of the workshop format aims to derive multi-disciplinary insights from individual contributions. Attempts will be made to draw analogies between input from different workshop participants and to derive potential synergies from them. The workshop chairs will document the topics raised on a white board or digital mind map.

Wrap-up (30min). The wrap-up session will be used to review and organise the notes collected during the discussion.

\section{TYPES OF CONTRIBUTIONS}

We call for two types of contributions for this workshop:

Short papers. Contributions describing original work in the area of interactive radio experiences. The work being described should have some technical background, and preferably contains evaluation results. The contribution should contain a discussion on who are the expected users of the solution, and how it fits in radio production workflows. Short papers should be submitted in ACM CHI Extended Abstracts Format with a length of up to 3,000 words.

Industry presentations. Contributions about work done at a broadcaster (or technology provider for radio). These contributions should focus on lessons learned, open challenges and future research work needed. Proposals for industry presentations are submitted as an abstract (also describing whether documentation of trials/demos could be shown), and contain a short CV of the presenter(s).

\section{CALL DISSEMINATION}

The workshop is supported by two ongoing H2020 projects: MARCONI (Multimedia and Augmented Radio Creation: Online, iNteractive, Individual ${ }^{2}$ ) and HRadio (Hybrid Radio everywhere for everyone ${ }^{3}$ ). In addition to publicising the call on social media channels of the organisers and their institutions, the following channels are used for distributing the call and soliciting contributions: 
${ }^{4}$ http://www.futurepulse.eu

${ }^{5}$ https://orpheus-audio.eu/

${ }^{6}$ https://tech.ebu.ch/

${ }^{7}$ https://www.ebu.ch/

${ }^{8} \mathrm{http} / / /$ www.aereurope.org/

${ }^{9}$ https://www.w3.org/community/audio-dev/

\section{Recent/ongoing research projects.}

- FuturePulse - Multimodal Predictive Analytics and Recommendation Services for the Music Industry ${ }^{4}$

- ORPHEUS - Object-Based Audio Experience ${ }^{5}$

Communities \& Organisations.

- EBU - The European Broadcasting Union's technical ${ }^{6}$ and editorial ${ }^{7}$ groups.

- AER - Association of European Radios ${ }^{8}$.

- Web Audio Community Group ${ }^{9}$ - Developers working on and with the Web Audio API.

- BBC - Practitioners and researchers at the BBC, as well as the BBC's domain-specific communities of academic partnerships: the audio, data-science and user-experience research partnerships.

- VRT - Innovation department involved in a wide range of research and innovation projects with both local and international partners.

- NEM - A community fostering innovation in media and creative industries consisting of more then 1,000 members.

\section{SHORT BIOS OF THE ORGANIZERS}

Rik Bauwens. is the lead developer at VRT Innovation. He obtained his MSc in Applied Engineering (Computer Science) in 2011. Rik is passionate about innovation in web technologies, new media and VR. In 2014, he joined VRT Innovation. He worked on the ICoSOLE project, which researches ways of enriching live experiences, as well as the ImmersiaTV project, which explored the very beginning of the already fascinating VR world. Currently, he leads the prototype team of VRT Innovation.

Hendrik Lievens. Hendrik is a Junior Researcher at Hasselt University, Belgium. His research interests are in computer networking and security, but also in virtual reality technology. Hendrik's master's thesis dealt with the topic of adaptive streaming of static light fields using the MPEG-DASH standard, the results of which will be published at ACM VRST 2018.

Maarten Wijnants. Maarten is a Senior Researcher at Hasselt University, Belgium. His primary research interest is in (adaptive) media streaming, originally from a technological but increasingly also from a user-centric perspective. Maarten's research has been published in - amongst others ACM Multimedia, Springer Multimedia Tools and Applications (MTAP), the Web conference (WWW) and ACM VRST. In 2017 and 2018, he has acted as associate chair for ACM TVX and was TPC member for ACM Multimedia. Maarten has also co-organized the 4th International Workshop on Interactive Content Consumption (WSICC) at ACM TVX 2016. 
Iris Jennes. Iris is user researcher at imec-SMIT. Her interests lie in combining insights in the strategies of commercial media actors with analysis of the tactics that users wield to gain more control over their experience. She is currently working on a $\mathrm{PhD}$ on innovative television advertising strategies and how these fit into the television audiencesâÁZ daily practices. In addition, Iris is involved in several research projects on hybrid radio, personalised news content and data management for online advertising.

Chris Pike. Chris leads the audio research team at BBC Research \& Development, UK, which focuses on personalised and immersive listening experiences. He leads the BBC Audio Research Partnership, through which the BBC collaborates with world-class universities, and is active in industry bodies, such as the ITU and the EBU. His work has seen the BBC using spatial audio with some of its biggest programmes, such as the BBC Proms, Planet Earth and Doctor Who. He is also a PhD candidate in the Audio Lab at the University of York, investigating the quality of binaural audio systems.

Werner Bailer. Werner is a Key Researcher at JOANNEUM RESEARCH's Connected Computing Group (Graz, Austria), with research interests in video content analysis, processing and retrieval and modelling of metadata of audiovisual content. He serves regularly as reviewer for journals (e.g., IEEE TIP, IEEE TCSVT and ACM ToMM) and conferences (e.g., ACM MM, IEEE ICME, ACM ICMR, MMM) Since 2012, he is co-organizer of the Video Browser Showdown interactive video search challenge, and has recently co-chaired workshops at ACM TVX and NIPS.

\section{REFERENCES}

[1] Rene Kaiser, Britta Meixner, and Joscha Jager. 2017. Reflecting on the Workshop on Interactive Content Consumption (WSICC) Series. IEEE MultiMedia 3 (2017), 83-88. 